Estrategias para potenciar el aprendizaje motor en el tratamiento vocal de las enfermedades neurodegenerativas

Strategies to ensure motor learning during the treatment of vocal problems in neurodegenerative diseases

Rosa Bermúdez de Alvear ${ }^{1}$, Ginés Martínez Arquero ${ }^{2}$

${ }^{1}$ Doctora en Medicina, Foniatra. Departamento de Radiología y Terapéutica Física, Oftalmología y Otorrinolaringología. Facultad de Medicina. Universidad de Málaga.

${ }^{2}$ Doctor en Medicina, Otorrinolaringólogo. Servicio de Otorrinolaringología. Hospital Regional Universitario Carlos Haya. Servicio Andaluz de Salud. Málaga.

Correspondencia:

Rosa Bermúdez de Alvear

Departamento de Radiología y Terapéutica Física, Oftalmología y Otorrinolaringología. Facultad de Medicina. Boulevard Pasteur ${ }^{\circ}$ 32. Campus de Teatinos. Universidad de Málaga. 29071-Málaga.

Tel. +34 605688490

Fax +34952131630

E-Mail: bermudez@uma.es, bermudezdealvear@gmail.com

Preprint del artículo publicado en: Revista de Logopedia, Foniatría y Audiología (2013)

33:13-24.

doi: 10.1016/j.rlfa.2012.07.004

Licencia Creative Commons CC BY-NC-SA 


\title{
Estrategias para potenciar el aprendizaje motor en el tratamiento vocal de las enfermedades neurodegenerativas
}

\begin{abstract}
Resumen:
Los déficits del sistema sensoriomotor tienen un efecto dramático sobre la calidad de vida de las personas con enfermedades neurodegenerativas, especialmente la afectación de los nervios craneales responsables de la deglución, fonoarticulación, expresión facial y voz. Actualmente existe evidencia científica de que al entrenar sistemáticamente los grupos musculares encargados de los movimientos fonorrespiratorios, puede mejorarse o lentificarse el avance de la enfermedad y sus efectos sobre el deterioro sensoriomotor. Sin embargo aún no se ha avanzado lo suficiente en el diseño de estrategias específicas para propiciar la generalización de los resultados terapéuticos, esto es, para favorecer la transferencia de las mejorías desde la clínica a la vida cotidiana del enfermo neurodegenerativo. En este estudio se exponen los principios básicos del aprendizaje motor, sus fases y pautas de administración, centrándose en la aplicación de estos aspectos al tratamiento de personas con problemas de voz de origen neurodegenerativo. En una segunda parte se hace una revisión de la literatura sobre los factores que potencian la efectividad del método LSVT ${ }^{\circledR}$. Se analiza cómo dicho modelo terapéutico aplica las teorías del aprendizaje motor al tratamiento de los problemas fonatorios de las personas que padecen Parkinson, proporcionando una generalización de las mejorías motoras y una repercusión de dichos beneficios a nivel de la lesión neurológica, la función vocal, la inteligibilidad del habla y la deglución, con resultados terapéuticos que han demostrado mantenerse a corto, medio y largo plazo por encima del nivel previo al tratamiento.
\end{abstract}

\begin{abstract}
:
Sensorimotor deficits have a devastating effect on quality of life of people with neurodegenerative diseases; and disruptions of craniofacial nerves are especially dramatic because they concern voice, speech, facial expression and deglutition. It has been scientifically evidenced that targeted training of laryngeal and respiratory muscle groups may reverse or slow disease progression as well as its cranial sensorimotor disorders. However there is still a lack of specifically designed strategies to achieve motor learning and to enhance the generalization of skill movements; e.g. there is a need for specific treatment protocols that improve the transfer of motor abilities from the clinical setting to the patient's actual environment. The purpose of this study is primarily focused on reviewing motor learning principles, its phases and ways of administration. In the second instance, the application of motor learning requisites to the treatment of vocal problems in neurodegenerative diseases is addressed; and accordingly literature about Lee Silverman Voice Treatment (LSVT $\left.{ }^{\circledR}\right)$ is further revised to analyze its efficacy. This method has been used with persons suffering from Parkinson disease and it has shown to improve the neurological lesion, vocal function, speech intelligibility and deglutition, with most of its effects being permanent in the short, medium and long term.
\end{abstract}

Palabras clave: aprendizaje motor, control motor del habla, eficacia terapéutica, enfermedad del Parkinson, generalización de resultados, intensidad vocal, enfermedades neurodegenerativas, Tratamiento Vocal Intensivo.

Key words: neurodegenerative diseases, Parkinson's disease, treatment efficacy, Lee Silverman Voice Treatment, speech motor control, voice loudness, motor learning, outcomes generalization. 


\section{Introducción}

Los déficits del sistema sensoriomotor tienen un efecto dramático sobre la calidad de vida de las personas con enfermedades neurodegenerativas, especialmente la afectación de los nervios craneales responsables de la deglución, fonoarticulación, expresión facial y voz. La severidad de los trastornos sensoriomotores es variable según cada tipo de enfermedad neurodegenerativa y cada estadío evolutivo. El Parkinson Idiopático (PI) es la más estudiada de todas, ya que no sólo es la que con mayor frecuencia conlleva alteraciones comunicativas y deglutorias, sino que al mismo tiempo es la que permite un mayor periodo de intervención al ser lentamente evolutiva. La hipofonía aparece en un $89 \%$ de los casos con PI; mientras que las alteraciones de la deglución suelen existir en un 75\%, pero al ser leves tan sólo un 20-41\% de los enfermos llega a referirlas y raramente son tratados por estos trastornos (Clarke et al., 1998). En la Esclerosis Lateral Amiotrófica (ELA) los síntomas iniciales varían según que la forma de presentación sea bulbar, medular o mixta bulbo-medular; pero en un $25 \%$ suele afectarse el habla desde el principio, produciendo una disartria mixta flácido-espástica y una disfagia de rápida evolución (Ramig et al., 1990). En estos casos la intervención para paliar el deterioro de la respiración, voz, habla y deglución es fundamental desde el momento del diagnóstico, ya que aproximadamente tras los dos primeros años de evolución, el tratamiento rehabilitador deja de ser eficaz y estas personas requieren ayudas técnicas para respirar, comunicarse y deambular, así como gastrostomía para ser alimentadas (Houd y Mangolds, 1999). En la Esclerosis Múltiple (EM) la disartria y la disfagia no son síntomas universales, suelen aparecer cuando la afectación motora es más severa y con una evolución más rápida (Beukelman et al., 1998). Se conoce que en un $40-51 \%$ de estos enfermos suele aparecer disartria, y en un 33\% disfagia desde el inicio de la enfermedad; pero ambos trastornos suelen ser de grado leve o moderado y únicamente un $2 \%$ de ellos refiere haber recibido tratamiento por ello. La disartria suele ser mixta, de tipo espástico-atáxico y generalmente produce una mayor alteración en los mecanismos del habla que de la voz (Hartelius y Lillvik, 2003). La Enfermedad de Huntington (EH), la Ataxia de Friedreich (AF) y la Atrofia Olivoponto-cerebelosa (AO) son enfermedades neurodegenerativa de carácter hereditario, cuya evolución es lentamente progresiva y afectan a las funciones sensoriomotoras como el habla, voz y deglución. La EH produce disartria de tipo hiperquinético por lesión extrapiramidal, mientras que las otras dos originan una disartria atáxica de origen cerebeloso y extracerebeloso. La aparición y severidad de estas alteraciones son variables según cada persona, pero al ir asociadas a déficits neuropsicológicos la rehabilitación se hace difícil (Beukelman et al., 1998).

A lo largo de las últimas tres décadas se han ido publicando estudios basados en la aplicación de los principios del aprendizaje motor al tratamiento de la disartria y apraxia del habla en niños y adultos con enfermedades neurológicas (Caruso y Strand, 1999; McNeil et al., 1997). Entre los métodos que han sido empleados para tratar los problemas de voz, habla y deglución en las enfermedades neurodegenerativas cabe destacar el denominado Tratamiento Vocal Intensivo o Lee Silverman Voice Treatment (LSVT®), que ha sido aplicado en el tratamiento del PI, el Parkinson asociado a atrofia muscular, algunas formas de Esclerosis Múltiple (EM), Esclerosis Lateral Amiotrófica (ELA) y las secuelas de accidentes cerebrovasculares (Mahler et al., 2009; Sapir et al., 2011).

Actualmente existe evidencia científica de que al entrenar sistemáticamente los movimientos fonorrespiratorios de la musculatura laríngea, orofaríngea y respiratoria mejora de forma estable la función vocal; incluso se han obtenido mejorías también en las otras funciones motoras de dichos grupos musculares, como la deglución, fonoarticulación y expresión facial (Narayana et al., 2010). Sin embargo aún no se ha avanzado lo suficiente en la utilización de estrategias específicamente diseñadas para propiciar la generalización de resultados terapéuticos, esto es, la transferencia desde la clínica a la vida cotidiana del enfermo neurodegenerativo (Fisher et al., 2008; Ransmayr, 2011).

A continuación haremos una revisión de los principios del aprendizaje motor cuya aplicación a la rehabilitación de la voz puede facilitar la generalización de los resultados terapéuticos. El objetivo es señalar las estrategias que en la literatura han demostrado favorecer la aparición de cambios relativamente permanentes en la actividad funcional del paciente con enfermedades neurodegenerativas y déficits sensoriomotores del sistema fonorrespiratorio. Finalmente expondremos los fundamentos del programa Entrenamiento Vocal Intensivo (Lee 
Silverman Voice Therapy, LSVT®) (Sapir et al., 2006) para resaltar los principios del aprendizaje motor que en este método se emplean.

\section{Fundamentos y etapas del aprendizaje motor}

El concepto de aprendizaje motor es definido como el proceso de adquisición de habilidades motoras coordinadas y precisas, a partir de una práctica sistemática y repetida que va generando una experiencia y hace que la conducta aprendida sea transferida de forma permanente a la actividad diaria del sujeto (McNeil et al., 1997). De este modo la habilidad motriz implicada en el habla, la voz o la deglución se convierte en una modificación relativamente estable y llega a ser utilizada de forma habitual.

El nivel de aprendizaje motor va a depender básicamente de las pautas de administración de las tareas de entrenamiento, aunque también son muy importantes las habilidades cognitivas del paciente, ya que la automatización va a depender en gran medida del grado de esfuerzo atencional y de la capacidad de almacenamiento de información que posea el enfermo. Esto no significa que una disminución leve o moderada de la atención y la memoria impida mejorar la disartria, la disfonía o la disfagia de una persona, pero sí implica que a medida que el deterioro de las capacidades cognitivas sea mayor, la recuperación del control motor va a ser más difícil y menos generalizable. Cuando la disfunción sensoriomotriz va asociada a déficits cognitivos y cambios de personalidad, la rehabilitación se hace difícil e incluso restringe mucho la potencial utilidad de las ayudas técnicas. De aquí se deduce la importancia de que la intervención se empiece lo más precozmente posible en todos los trastornos neurodegenerativos (Beukelman et al., 1998; Square, 1999).

En presencia de déficits cognitivos leves, la programación y las pautas de administración de los protocolos terapéuticos se convierten en los factores más determinantes para que lograr el aprendizaje motor.

En la literatura sobre este tema se distinguen dos fases en la programación del tratamiento: la pre-práctica y la práctica. Respecto a la fase pre-práctica existe una serie de factores que actúan como pre-requisitos del aprendizaje, pues motivan al paciente y lo preparan para realizar la práctica que posteriormente se le va a solicitar (McNeil et al., 1997).

El primer precepto a considerar es crear motivación hacia el aprendizaje, y la mejor forma de hacerlo es asegurándose de que el paciente comprenda por qué una determinada tarea es útil dentro del programa de tratamiento vocal. Para ello se le deben ofrecer ejemplos cotidianos que reflejen la utilidad práctica de las funciones que va a entrenar en la clínica; incluso se le debe hacer partícipe en la planificación de los objetivos preguntándole qué es lo más importante que espera conseguir con el tratamiento. El clínico debe conocer las expectativas del enfermo y sus familiares para que siempre que sea posible, pueda darle prioridad al entrenamiento de esas funciones que subjetivamente son valoradas como más necesarias. Es a partir de estas demandas y sobre la base de estos intereses subjetivos de la persona enferma y su entorno cercano, que el clínico va a poder explicarles los fundamentos del tratamiento, las posibles limitaciones que puedan existir para conseguir los objetivos y la eficacia de las tareas que realmente pueden funcionar para lograr una mejoría. Mientras mejor sea la colaboración entre el clínico y los afectados para determinar la jerarquía de prioridades y los medios para conseguirlas, mayor será su motivación e implicación en el proceso de recuperación (Strand y Skinder, 1999).

Como parte de los requisitos de la pre-práctica es asimismo importante asegurarse de que el individuo enfermo comprende lo que se le va a pedir en cada ejercicio concreto que se le plantee, para lo cual se deben emplear unos instantes, antes de cada ejercicio, para describirle las líneas generales de lo que se le va a solicitar a continuación. Las explicaciones que se le den han de ser sencillas, con un vocabulario concreto y sin tecnicismos. Al principio bastará con que se le expliquen uno o dos aspectos del movimiento que vaya a ejecutar, los principales para empezar a practicarlo; luego se le irán dando más detalles, pero inicialmente no se debe sobreexplicar para no confundirle.

Otro aspecto significativo de la pre-práctica es que se le dé preeminencia al aprendizaje mediante la observación, de modo que la exposición del modelo motor que ha de imitar sea más visual que verbal. Esto es especialmente importante en pacientes con falta de atención o déficits de comprensión verbal y se puede lograr mediante distintos tipos de estrategias: el clínico puede ejecutar la secuencia motriz frente a él, o bien enseñarle material gráfico como imágenes 
anatómicas simplificadas o videos de otros pacientes realizando la tarea. Asimismo será útil permitirle observar in vivo la actuación de otra persona afecta, pues estar junto a un compañero es generalmente una de las formas más motivadoras para cualquier enfermo neurodegenerativo. El entrenamiento en grupo de dos o tres sujetos con el mismo problema es por ello bastante beneficioso, siempre y cuando el estadío evolutivo de todos así lo permita.

Durante la pre-práctica de cada ejercicio también se deben establecer unas referencias claras respecto a lo que es correcto o no en una ejecución concreta, de modo que el sujeto sepa autovalorarse. Se ha demostrado que el hecho de que el enfermo conozca con claridad cómo esperamos que sea su ejecución, tiene una gran relevancia para incentivarlo y enseñarle a ser autónomo. Evitemos decirle "haga lo mejor que pueda"; en cambio, establezcamos previamente con él un nivel específico a alcanzar durante la tarea, planteándole hasta dónde ha de llegar en cada parámetro del ejercicio; es decir, propongámosle una meta clara y asequible para él en ese momento y ayudémosle a que sepa de antemano hasta dónde debe esforzarse. Por ejemplo, al solicitarle que emita una vocal, se la reproduciremos e incluso se la compararemos con otra vocal de igual timbre y distinto tono, intensidad o duración; con esto adquirirá referencias nítidas de lo que es y no es correcto en ese momento.

La fase de práctica o entrenamiento motor es la más importante del aprendizaje, ya que es el proceso durante el cual se practica la tarea motora y se retiene. Durante este periodo se internaliza la organización temporal y espacial de los movimientos, se automatizan las secuencias y se generan los programas motores cerebrales (Square, 1999). En relación a esta fase, la literatura sobre aprendizaje motor establece dos sub-etapas básicas: a) la etapa de adquisición, que es el periodo durante el cual se aprende a ejecutar la tarea sin errores, mediante la repetición sistemática y con la ayuda del clínico; y b) la etapa de retención, que es un periodo posterior, cuando en ausencia de una guía o modelo, la tarea puede ser evocada y realizada sin errores, fuera y dentro de la clínica. Lograr la retención de la tarea implica que se ha alcanzado el aprendizaje motor y que la habilidad puede ser generalizada a otros contextos (Strand y Skinder, 1999).

Es muy importante distinguir entre estos dos estadíos, pues cada uno tiene sus propios requisitos de aprendizaje, de modo que los factores que facilitan la adquisición, no suelen favorecer la retención y viceversa. Por ejemplo, cometer pocos errores durante la fase de adiestramiento inicial no siempre implica que el paciente haya aprendido a ejecutarla por sí solo o que sea capaz de recordarla al cabo de unos días. En otras palabras, durante la práctica, las pautas de entrenamiento que favorecen la ejecución sin errores o la adquisición, no suelen ser eficaces para favorecer la retención de la tarea y su evocación a largo plazo. De ahí la importancia que tiene saber analizar los factores que están favoreciendo cada etapa del aprendizaje.

\section{Pautas de administración de las tareas}

Para que durante la práctica de una habilidad se cumplan estos dos estadíos del aprendizaje, la adquisición y retención del movimiento diana, es preciso llevar a cabo una planificación específica, tanto de los ejercicios de entrenamiento como de la pauta para proporcionar el feedback externo.

El hecho de que la programación de las tareas sea específica para generar aprendizaje, implica establecer una pauta temporal de administración de las sesiones, así como una serie de estrategias para la repetición sistemática de las tareas. Entre el conjunto de factores a tomar en consideración para programar el tratamiento son de resaltar los cuatro siguientes: la frecuencia y duración de las sesiones; la combinación de objetivos únicos o múltiples en cada tarea; la forma de repetir y combinar las tareas durante las sesiones; el tipo de feedback y la frecuencia con que éste se le ofrezca al paciente durante la práctica.

Respecto a la distribución temporal de las sesiones de rehabilitación de las habilidades motoras comunicacionales, la pauta más recomendada por la opinión de expertos consiste en organizar sesiones frecuentes. La duración de éstas puede ser variable, pero deben ser lo suficientemente largas como para permitir una práctica repetida de cada tarea (Strand y Skinder, 1999). Por ejemplo, si contamos con dos horas semanales de tratamiento, parece más recomendable distribuir las dos horas en cuatro sesiones de media hora (programación distribuida), que impartir dos sesiones semanales de una hora cada una (programación masiva). 
Una cuestión sin resolver científicamente se refiere al número de pacientes en cada sesión; las sesiones individuales favorecen un mayor número de repeticiones, por lo que en este sentido fomentan más el aprendizaje motor en general. Sin embargo la práctica junto a un compañero parece incentivar más el aprendizaje a través de la motivación y la observación (McNeil et al., 1997). Es por lo tanto la capacidad y la actitud de cada paciente lo que nos debe guiar al respecto.

En lo que concierne a la simplicidad o multiplicidad de los objetivos de cada tarea, se ha visto que el entrenamiento mediante tareas de un único objetivo favorece la disminución de los errores durante la práctica, ya que al repetir una tarea sencilla, progresivamente se van cometiendo menos errores y se va favoreciendo la adquisición del aprendizaje. Un ejemplo de objetivo único sería repetir una secuencia de sílabas breves cuidando sólo de que estén apoyadas con una contracción abdominal, sin dar instrucciones sobre tono ni intensidad. Las tareas de objetivo único se recomiendan en pacientes severamente afectados, como fase inicial del tratamiento (Strand y Skinder, 1999).

Por el contrario, la combinación de varios objetivos dentro de una misma tarea favorece la retención o automatización del aprendizaje en mayor medida que cuando se estimula sólo un componente aislado del movimiento (Strand y Skinder, 1999). Una tarea de objetivos múltiples implica que durante cada ejecución el paciente aprende a controlar más de un aspecto del sistema comunicativo, por ejemplo la postura y la respiración; o bien estas dos variables junto a otras dos como el control de la emisión laríngea y la resonancia. Un ejemplo de tarea con objetivos múltiples sería emitir una vocal de forma sostenida sin variar el tono predeterminado, durante el máximo tiempo y a máxima intensidad. Otro ejemplo de mayor complejidad sería si además se le indica que debe controlar la postura y hacer una pausa inspiratoria al principio y otra espiratoria al final de cada emisión, ya que así se estaría trabajando para automatizar el uso coordinado de todo el esquema corporal vocal con el fin de elevar la intensidad de la voz. La multiplicidad de objetivos favorece la retención y generalización del control motor, pero ha de ser administrada en función de la capacidad de aprendizaje del paciente y de forma paulatina, pues a medida que va adquiriendo una habilidad motora (apoyar la emisión de un tono, por ejemplo) se pueden ir introduciendo más objetivos en cada secuencia, induciéndole así a autocontrolar más parámetros.

Respecto a la programación de las tareas dentro de una misma sesión, está claro que la práctica ha de basarse en la repetición sistemática para fomentar el aprendizaje motor. Se ha demostrado que mientras mayor sea el número de repeticiones, mayor nivel de adquisición y retención se logrará (McNeil et al., 1997). Para combinar la repetición de tareas y de objetivos dentro de cada sesión se pueden hacer dos tipos de programaciones, bien una práctica en bloque o bien una práctica randomizada.

La práctica en bloque consiste en que todos los ejercicios dirigidos al mismo objetivo se practican juntos, sin que haya interrupciones ni distracciones, antes de pasar al siguiente objetivo. Cuando las tareas se organizan en bloques de objetivos, parece favorecerse la fase de adquisición del aprendizaje, pues así disminuyen los errores de la ejecución. Por ejemplo, si en una tarea fonatoria se pretende trabajar un único objetivo, se dedicará el tiempo sólo a incrementar dicho fin (el tiempo fonatorio, por ejemplo: hasta dos segundos, cuatro, seis, etc.). Si se persiguen dos objetivos en bloque (como el aumento de la duración y del tono, por ejemplo), se dedicará la primera parte de la sesión a trabajar uno de ellos (el aumento del tiempo fonatorio, con vocalizaciones sostenidas de mayor duración cada vez); mientras que en una segunda parte de la sesión se estimulará el siguiente objetivo (la subida de dos semitonos mediante secuencias de vocales breves). Con el fin de no crear un acostumbramiento o sobrehabituación a la tarea practicada en bloque, dentro de una misma sesión se van cambiando los tipos de estímulos (variando el segmento verbal, que pueden ser vocales, sílabas, bisílabos, etc.).

Cuando la práctica es combinada de forma aleatoria o randomizada, implica que la presentación de los diferentes objetivos se va intercalando a lo largo de la sesión sin llevar un orden fijo o invariable. La práctica aleatoria favorece más la retención y la generalización que la práctica en bloques, ya que se aproxima más a las condiciones reales del habla porque le exige coordinar los diversos sub-sistemas de forma variable (postural, respiratorio y fonatorio, que asimismo incluye emisión y resonancia). La variedad de tareas puede ser tan extensa como la imaginación del clínico y las necesidades del paciente. Veamos algunos ejemplos: una práctica randomizada con un solo objetivo (la variación tonal) sería hacer secuencias con la misma 
vocal, pero variando el tono aleatoriamente en cada emisión. Una combinación aleatoria con tres objetivos distintos consistiría, por ejemplo, en trabajar tres grupos musculares como el lingual, laríngeo y costodiafragmático, variando el rango de contracción lingual, la frecuencia de vibración glótica y la duración de las emisiones en cada secuencia: una secuencia puede estar formada por tres vocales de distinta elevación lingual (alta anterior, alta posterior, plana), sostenidas tres segundos en un tono cómodo; a continuación otra secuencia de esas vocales en tono agudo y con duración más breve; seguida de otra secuencia de dichas vocales en tono grave y duración más sostenida; y así sucesivamente. Otro ejercicio similar de práctica aleatoria, pero añadiendo un objetivo y un grupo muscular más, podría consistir en controlar el tiempo para hacer las pausas inspiratorias antes de cada secuencia.

Evidentemente la programación de las tareas y la complejidad de los objetivos han de venir determinados por la capacidad de respuesta del paciente, el tipo de alteración y el grado de severidad de la lesión. Los casos más complicados se beneficiarán de un entrenamiento con tareas de objetivo único que se repitan en bloque, para favorecer así el aprendizaje en sus fases iniciales y disminuir el número de errores en los movimientos aislados y simples; será por tanto la primera elección en enfermos neurodegenerativos con lesiones severas (Kleinow et al., 2001; Sapir et al., 2002). Pero a medida que ganen autocontrol motor, se les podrán ir introduciendo tareas con objetivos múltiples y programaciones randomizadas, ya que ello facilitará más la retención de habilidades verbales complejas que reflejen los movimientos del habla natural (Adams y Page, 2000).

\section{Estrategias de feedback o retroalimentación de la información}

Durante el tratamiento de un enfermo neurodegenerativo con trastornos sensorio-motores las técnicas para proporcionar feedback son esenciales para potenciarle la conciencia del movimiento, ya que le aportan una información sensorial que no le llega por las vías naturales de retroalimentación (Adams y Page, 2000; Schulz et al., 2000). Con estas estrategias se le va a facilitar una mejor comprensión de la tarea y una más pronta ejecución sin errores (adquisición); asimismo también se le ayudará a ganar autonomía y automatización (retención). Dicha retroalimentación sensorial puede ser administrada por muy diversos medios, pero básicamente se puede optar por dos vías de aplicación, según se empleen instrumentos electrónicos o según sea el clínico quien le proporcione el feedback verbalmente, siendo ambas vías complementarias, no excluyentes.

Feedback instrumental. Hay publicados trabajos en los que se muestran los efectos conseguidos mediante diversos tipos de tecnología como el electromiograma (Gentile et al., 1994); sistemas digitalizados de análisis acústico de la voz o de estimulación auditiva (Kiran et al., 2001); los transductores de presión subglótica (Thompson-Ward et al., 1997); o incluso otros instrumentos tan sencillos como el cronómetro (Ramig et al., 1995). Mediante estos tipos de feedback instrumental se le ofrece al paciente información instantánea sobre los parámetros fisiológicos que están bajo control neurológico, tales como la fuerza, la duración, la intensidad, la altura tonal, la presión subglótica, etc.

Feedback clínico. De todas las pautas que podemos emplear para fomentar el aprendizaje, quizás la más efectiva sea la información que le proporciona el clínico al paciente para mostrarle explícitamente la calidad que ha logrado en el ejercicio (Fox et al., 2002, 2006). La información aportada puede ser de dos tipos: bien un conocimiento sobre la ejecución o bien un conocimiento sobre el resultado global. En el primer modelo, al acabar la tarea se le informa sobre cómo ha conseguido determinados parámetros físicos como la postura, la velocidad, la fuerza, el tono o la intensidad, esto es, se analizan los distintos componentes de la ejecución. En el segundo tipo de feedback, al acabar se le comunica si ha conseguido, o no, el objetivo general preestablecido, pero sin entrar en detalles; por ejemplo, se le dice "sí, eso es", o bien "esto no está del todo logrado”.

A la hora de reforzar a la persona enferma, el clínico debe respetar unas pautas temporales, ya que los procesos de aprendizaje requieren un tempo, esto es, el sujeto debe adquirir un ritmo bien diferenciado de acción-movimiento y de no-acción o reposo. De este modo, al acabar una tarea se le deben proporcionar unos instantes de silencio para que su cerebro disponga del tiempo necesario para hacer consciente su propia información sensoriomotriz sobre el movimiento que ha ejecutado. Igualmente, antes de volver a comenzar la siguiente tarea, debe 
tener unos instantes para programar la siguiente secuencia. Es por esto que siempre se debe dejar un intervalo de latencia previo al feedback, y así el clínico esperará unos instantes, tras la finalización del ejercicio, antes de darle la retroalimentación. Esta latencia debe ser breve (uno o dos segundos), si es demasiado larga se disminuye el aprendizaje; y si por el contrario, estos instantes se ocupan con una actividad extraña a la tarea, como conversar o moverse, el potencial aprendizaje motor que podría haberse obtenido, se inhibe por completo. Por otro lado, como después de recibir el feedback el paciente necesita hacer consciente la nueva información obtenida, es necesario dejar también otro intervalo de latencia posterior al feedback. Aproximadamente con unos dos o tres segundos de parada y silencio es suficiente para ello y para planificar el siguiente ejercicio.

Otro aspecto temporal importante que se debe tomar en consideración respecto al feedback, es la frecuencia con que éste se ofrece (Adams y Page, 2000). Puede ser un feedback inmediato, impartido tras cada respuesta; o bien un feedback retardado, también llamado sumatorio, pues se le informa al cabo de varias ejecuciones seguidas. Parece ser que el feedback inmediato facilita la adquisición de habilidades motoras nuevas, es decir, le ayuda a disminuir los errores durante la práctica del ejercicio; pero sin embargo no parece favorecer la retención del aprendizaje. Al contrario de lo que comúnmente se cree, el feedback inmediato puede que le aporte al enfermo una excesiva cantidad de información y por esto le dificulte la memorización del ejercicio. Por el contrario, el feedback retardado o sumatorio parece ser más eficaz para fomentar la retención y automatización del aprendizaje, pues hace recaer una mayor responsabilidad sobre el paciente para autovalorar su ejecución. Cuando la tarea sea fácil, el feedback se puede retardar bastante, por ejemplo se puede impartir tras unas 15 ejecuciones; pero si la tarea es compleja o el paciente tiene limitaciones cognitivas, el feedback debe ser inmediato o darse con poco retardo (por ejemplo cada tres o cinco respuestas). Para pacientes con daño neurológico, comenzar el tratamiento dándoles un feedback sumatorio puede ser muy difícil para ellos; una opción puede ser comenzar por impartirlo de forma inmediata, luego cada tres respuestas y así ir retardándolo a medida que el paciente va ganando habilidad (Strand y Skinder, 1999).

\section{Otros factores condicionantes de la retención y automatización del aprendizaje motor}

Para permitir que determinado movimiento sea adquirido y sobre todo automatizado por el sujeto con trastornos neurodegenerativos, se deben considerar dos tipos de factores condicionantes del aprendizaje, imprescindibles para facilitar su transferencia a la actividad diaria del paciente. En primer lugar, las condiciones biofísicas del movimiento que se entrene han de ser idénticas a las del movimiento diana. Es lo que se ha dado en llamar "targeted training”, lo cual implica que los movimientos practicados en la clínica han de reproducir los mismos rasgos motrices en cuanto a postura, fuerza, amplitud, dirección y velocidad, que posea la habilidad que se pretenda alcanzar con la rehabilitación (Russel et al., 2010). El segundo gran conjunto de factores que favorecen la retención del aprendizaje motor en la fase práctica está dirigido a facilitar el desarrollo de un nuevo esquema corporal o imagen mental del movimiento en el sujeto con una enfermedad neurodegenerativa. A continuación se analizan estos dos tipos de requisitos del aprendizaje motor.

Condiciones biofísicas de la ejecución del movimiento. La tarea que se practique debe ser lo más similar posible al movimiento diana que se desee estimular. Estas condiciones se refieren a la postura y a los parámetros físicos del movimiento en cuestión: fuerza, amplitud, velocidad, dirección y precisión (Square, 1999). La postura ha de ser facilitadora del movimiento, pues se debe ajustar de tal forma que facilite la actuación del grupo muscular adecuado e inhiba la intervención de otros grupos musculares sinérgicos o compensatorios del déficit. Así, al inmovilizar los músculos que actúan de forma sincinésica a los músculos del grupo diana, impediremos que los primeros participen en la tarea y le resten actividad a los segundos. Por ejemplo, si se evita la extensión del cuello durante la emisión de agudos, se favorece una mejor contracción del cricotiroideo; o si se disminuye la contracción de bandas ventriculares en la emisión sostenida de vocales, se facilita la ondulación de los repliegues vocales. Por otro lado, el movimiento practicado ha de poseer los mismos rasgos físicos que son específicos de la habilidad motora que se pretenda rehabilitar. Por ejemplo, el entrenamiento de la contracción de la pared abdominal mediante tareas no-verbales o la ejecución del soplo áfono, son ejercicios 
con muy poca o ninguna repercusión en la adquisición y retención de un mayor apoyo respiratorio de la voz, pues con estas tareas no-fonatorias se está realizando un movimiento independiente del cierre glótico fonatorio y que posee características físicas distintas al soplo fonatorio en cuanto a fuerza, amplitud, velocidad de contracción, etc. En definitiva, no existe evidencia científica de que los ejercicios no-fonatorios favorezcan el desarrollo de movimientos fonatorios, por lo tanto no son útiles para retener al aprendizaje.

Para que el movimiento practicado tenga los mismos rasgos físicos que el movimiento fonatorio diana, se deben practicar tareas con los mismos estímulos verbales que se pretendan adquirir y automatizar. En otras palabras, al diseñar un ejercicio de adiestramiento debemos cuidar que el tipo de contracción muscular, el grado de fuerza, la dirección y la velocidad del movimiento, reproduzcan las mismas condiciones biofísicas que tiene el movimiento natural (Russel et al., 2010).

El tipo de contracción laríngeo es otro rasgo físico a tener en consideración. Puede ser una contracción concéntrica (el músculo se acorta al contraerse, como el tiroaritenoideo al emitir un grave) o excéntrica (el tiroaritenoideo se alarga, por acción del cricotiroideo y la musculatura intrínseca, al emitir un agudo). Si el objetivo perseguido es incrementar la fuerza de la musculatura de cierre laríngeo, se deben realizar ejercicios isométricos, durante los cuales los grupos musculares no varían su longitud, pero en cada ejercicio se podrá ir variando dicha longitud y aumentando la duración o la fuerza de la contracción. Cada tipo de contracción se podrá trabajar aisladamente o en secuencia, según se quiera estimular la adquisición o la retención de ese objetivo. Por ejemplo: para aumentar el cierre glótico (fase de adquisición) es eficaz mantener la contracción isométrica de la musculatura laríngea durante una vocal sostenida, sin variar su tono ni intensidad en el mismo ejercicio. Pero sin embargo, si se pretende estimular la automatización de dicho cierre glótico (fase de retención), se combinarán vocales de distinto tono y duración en una misma secuencia, es decir, se coordinarán contracciones concéntricas (notas graves) y excéntricas (agudos) dentro de la secuencia.

El grado de fuerza ha de ser igualmente específico para cada objetivo motor. Pero en el caso de las enfermedades neurodegenerativas, si se persigue disminuir la debilidad muscular de la voz, es necesario incrementar la fuerza con que se realiza cada tarea fonatoria, realizándola con máximo esfuerzo, incluso contra resistencia.

La velocidad del movimiento que entrenemos en la práctica clínica debe ser la misma que la del movimiento fonatorio natural que el enfermo necesita recuperar. Un movimiento laríngeo fonatorio depende de la amplitud y de la frecuencia de vibración glótica, que a su vez dependen de la tensión glótica y de la presión subglótica, es decir, del flujo aéreo espiratorio y de la tensión muscular de la musculatura laríngea (Scherer, 1991). Entonces, al entrenar la fonación en una enfermedad neurodegenerativa, debemos planificar objetivos para ir estimulando progresivamente todo el rango tonal del paciente a intensidades que el paciente pueda emitir; es así como se conseguirá estimular todos los movimientos glóticos que el sujeto pueda emplear en el habla.

Es también importante que en las tareas fonatorias se estimule el mismo ritmo de ejecución que se lleva en el habla espontánea, para lo cual el objetivo será instaurar el ritmo que viene marcado por la coordinación fonorrespiratoria. Para esto se deberá practicar un patrón de pausas inspiratorias frecuentes, pero que marquen un ritmo fonorrespiratorio cómodo y natural (Kleinow et al., 2001; Sapir et al., 2006). En la literatura existen discrepancias respecto a la conveniencia de modificar el ritmo del habla de los sujetos disártricos para mejorar su inteligibilidad; en general los resultados publicados vienen a indicar que variar el ritmo del habla no parece ser un buen objetivo terapéutico. Esto es debido a que las alteraciones del ritmo no ejercen una repercusión directa ni constante sobre la inteligibilidad: ni la lentificación ni la aceleración se asocian a cambios lineales en la inteligibilidad (Kleinow et al., 2001; Neel, 2009). Estos cambios de ritmo pueden incluso empeorarla, pues provocan variaciones impredecibles en la duración los segmentos acústicos, los cuales se reorganizan sin seguir un patrón lineal ni proporcional entre ellos, distorsionando así la inteligibilidad. Por ejemplo, al acelerar el habla se acorta más la duración de las vocales que de las consonantes, mientras que apenas aumenta la amplitud de los movimientos labiales (Weismer et al., 2000). Por otro lado se ha evidenciado que al enlentecer el ritmo aumenta la inestabilidad en la voz del enfermo con PI (Kleinow et al., 2001). 
Desarrollo de una nueva autoconciencia del movimiento. Finalmente, para que la práctica motriz llegue a ser automatizada, se debe también estimular la representación de una nueva imagen mental del movimiento. Para esto es útil el empleo de dos tipos de caminos o recursos: por un lado el empleo de estrategias para que el sujeto lleve a cabo una práctica mental a solas; por otro, la utilización de las técnicas de feedback o retroalimentación externa, que son recursos para proporcionarle al sujeto la mayor cantidad posible de información sensorial sobre el movimiento que esté realizando o que acabe de terminar, como ya fue comentado anteriormente

La práctica mental favorece la retención del aprendizaje (McNeil et al., 1997) siempre y cuando se le den instrucciones al paciente para la práctica sistemática de la tarea motora a solas, en un lugar relajado y silencioso, donde se pueda concentrar en imaginar la trayectoria y la fuerza del movimiento sin distracciones, visualizando mentalmente cómo el movimiento se desarrolla en tiempo real, aunque él no sea aún capaz de realizarlo corporalmente. Si la capacidad de concentración es suficiente, es positivo visualizar mentalmente la secuencia motora porque al visualizarse a sí mismo realizándola con éxito y escuchar mentalmente el sonido que produce, se incrementa el feedback interno y la imagen mental del movimiento. En caso de que el paciente ya sea capaz de llevar a cabo físicamente y por sí mismo la tarea que esté entrenando, la práctica a solas va a evocar y hacer conscientes las sensaciones propioceptivas y auditivas. Todo ello refuerza el esquema motor porque lo nutre de más información sensorial y ayuda a los circuitos tálamo-corticales a retener una imagen mental kinestésica.

\section{Aplicación de los principios del aprendizaje motor en el Tratamiento Vocal Intensivo (LSVT®)}

Este método fue específicamente desarrollado para mejorar la hipofonía de la disartria hipokinética del PI y consiste en la integración de otras técnicas ya conocidas en la rehabilitación motriz de extremidades, junto con las ya utilizadas en intervención vocal. Sus autores han demostrado cambios positivos tanto a nivel de la lesión neurológica central (Narayana et al., 2010), como de las limitaciones funcionales de los órganos periféricos (Fox et al., 2006), evidenciando también el mantenimiento de los resultados a corto, medio y largo plazo (Ramig et al., 2001a; Sapir et al., 2002, 2011). Incluso se ha encontrado que sus efectos se generalizan mejorando las disfunciones motrices de otros subsistemas como el fonoarticulatorio, deglutorio y la musculatura facial de enfermos neurodegenerativos (Sapir et al., 2006).

Aunque ha supuesto un avance muy importante en el tratamiento de las enfermedades neurodegenerativas, no sólo del PI, es un programa que resulta complicado de llevar a la práctica, tanto para los clínicos como para los pacientes, pues sigue una pauta muy intensiva y requiere sesiones largas y frecuentes, durante aproximadamente dos meses y medio. Es por esto que se están desarrollando nuevas modalidades de aplicación del Tratamiento Vocal Intensivo mediante videoconferencia y se están demostrando resultados similares a las sesiones presenciales (Howell et al., 2009; Constantinescu et al., 2011).

El mecanismo fisiopatológico del PI se explica por la disminución de la población de neuronas dopaminérgicas en la sustancia negra, es decir, por la disminución del neurotransmisor dopamina, que se puede considerar responsable de amplificar el impulso motor que llega a la corteza y al mismo tiempo de regular su velocidad y precisión. Consecuentemente el estímulo motor que desde las áreas motoras corticales llega a la musculatura laríngea y respiratoria se debilita en el PI. Adicionalmente se produce también un déficit dopaminérgico en el sistema pálido-estriado, lo que provoca un enlentecimiento del procesamiento de información sensorial o feedback inetrno, lo cual acarrea un déficit en el autocontrol de los actos motores durante su ejecución. Es decir, el sujeto no es consciente de que su voz es débil, ni tampoco de que va debilitándose progresivamente si él no hace un esfuerzo constante.

Esquemáticamente, se suponen tres tipos de mecanismos subyacentes al trastorno de voz del PI: a) la existencia de una reducida amplitud en el impulso motor hacia los músculos respiratorios, laríngeos y orofaciales; b) la dificultad para iniciar y mantener el movimiento, es decir, para iniciar la fonación con un buen cierre glótico y presión subglótica, así como para mantener estos parámetros hasta el final de la emisión; c) además aparece una falta de autocontrol de la fonación debido a los déficits sensoriales en el sistema pálido-estriado, que es 
uno de los centros donde la información sensorial es utilizada para monitorizar y controlar la ejecución del movimiento. En otras palabras, por un lado existe un impulso motor de amplitud débil hacia los músculos fonorrespiratorios, laríngeos y orofaciales; por otro, los déficits sensoriales le impiden al sujeto ser consciente de esta debilidad vocal y monitorizar el resultado acústico de su esfuerzo fonatorio.

Estos mecanismos fisiopatológicos se manifiestan en el PI mediante signos de rigidez muscular, hipoquinesia, bradiquinesia y temblor; afectando a los movimientos de extremidades tanto como a los de la musculatura orofacial, faringolaríngea y costo-diafragmática. Como consecuencia, el enfermo posee dificultad para iniciar la emisión de voz, que es poco audible desde su comienzo porque tanto la presión subglótica como el cierre glótico son débiles; y además este volumen va disminuyendo progresivamente durante la emisión. La voz se caracteriza entonces por la debilidad, monotonía y timbre ronco con escapes aéreos. A esto se le suma la falta de información sensorial, que le impide al sujeto ser consciente de esta baja intensidad de voz y controlarla (Mid et al., 2008). Estos datos tienen gran importancia clínica, pues indican la necesidad de potenciar la información sensorial del enfermo con PI como una forma de tratamiento específico para sus alteraciones motoras, pues si no reciben esta información no son capaces de reconocer el nivel de intensidad que usan, ni el nivel de esfuerzo que han de emplear para aumentarla y mantenerla audible.

La hipótesis sobre la que se construyó el Tratamiento Vocal Intensivo considera que la intensidad de voz del enfermo con PI puede incrementarse si se logra incrementar la amplitud del estímulo que llega a la musculatura fonorrespiratoria, ya que el mecanismo subyacente a esta hipofonía es la disminución del impulso motor que parte desde los circuitos nigro-estriados y llega hasta la musculatura laríngea y respiratoria. Es por ello por lo que inicialmente el objetivo del tratamiento fue incrementar el input que llega a la musculatura laríngea aumentando el esfuerzo fonorrespiratorio. Posteriormente el método se ha ido perfeccionando e incorporando un mayor énfasis en las técnicas de feedback sensorial mediante claves internas y referencias externas, de modo que no sólo se entrene al paciente en incrementar la intensidad y el tono vocal, sino que también se le adiestre en reconocer el esfuerzo necesario para mantenerla en dicho nivel (Fox et al., 2002; Spielman et al., 2007).

Frente a este cortejo de alteraciones, la eficacia del método LSVT radica en su metodología para planificar los objetivos, programar las tareas terapéuticas y emplear el feedback sensorial externo. En otras palabras, dirige sus objetivos hacia la compensación de los mecanismos fisiopatológicos de la enfermedad, programa las tareas según los principios del aprendizaje motor para adaptarlas al déficit cognitivo leve, y estimula la creación de una nueva imagen mental del movimiento en estos sujetos. Seguidamente se analizan estos aspectos.

Los objetivos del LSVT se centran en elevar la intensidad vocal mediante el incremento del cierre glótico y del input respiratorio. En segundo lugar también se estimulan otras metas terapéuticas como son el aumento del tiempo fonatorio máximo, el rango tonal, la variabilidad de la frecuencia fundamental y la coordinación fonorrespiratoria. El tercer grupo de objetivos consiste hacer evidente y potenciar en el sujeto la autopercepción del esfuerzo que está realizando cuando está emitiendo una voz de intensidad normal. Como ya ha sido comentado, esto último se lleva a cabo mediante técnicas de feedback, que le van entrenando a calibrar y a mantener el mismo esfuerzo fonatorio a pesar de no ser consciente del resultado acústico que tiene su voz (Liotti, 2003).

Estos objetivos se trabajan instruyendo al paciente siempre con la consigna "hable fuerte, lo más fuerte que pueda, y con el máximo esfuerzo", o bien "piense: fuerte; y luego hable fuerte. Asimismo se emplean protocolos de ejercicios específicos. Para maximizar el cierre glótico se incluyen ejercicios de contracción isométrica de laringe (cierre valvular), y se le pide que haga una fonación a máxima intensidad y durante el máximo tiempo posible. Posteriormente se repite la misma consigna y modalidad de ejercicios en distintos tonos del rango tonal, hasta llegar a los extremos agudo y grave. La coordinación fonorrespiratoria se estimula instruyendo constantemente al paciente para que realice inspiraciones profundas antes de empezar a hablar fuerte, "hable siempre con los pulmones llenos de aire", así como para que haga pausas respiratorias muy frecuentes. Los tipos de estímulos verbales se van variando para presentarlos jerarquizados según su complejidad: desde la repetición de vocales simples, hasta la lectura y la conversación espontánea, pasando por segmentos de creciente longitud. 
La programación de tareas prácticas sigue una sistemática consistente básicamente en la repetición de tareas en bloque, que van dirigidas a producir un único objetivo por vez, especialmente aumentar el cierre glótico con un alto nivel de esfuerzo y una gran presión de aire subglótico. Los ejercicios del entrenamiento están diseñados para que sean simples y no impliquen movimientos complejos ni simultáneos, esto es, son secuencias en bloque de un mismo movimiento realizado con máximo esfuerzo (Spielman et al., 2007).

El programa le da mucha importancia al desarrollo de una nueva autoconciencia del movimiento, la cual se estimula mediante técnicas de feedback sensorial (auditivo, visual y propioceptivo), la práctica en solitario y la constante motivación del enfermo hacia las tareas (Ramig et al., 2001b; Sapir et al., 2003). El clínico le proporciona al paciente un feedback verbal constante, consistente en explicarle cómo lo ha hecho al final de cada tarea, en medir los resultados de forma objetiva y en reproducirle su propia voz grabada en audio y video. Se utilizan grabaciones para enseñarle a sopesar su esfuerzo fonatorio según la calidad de la voz que haya resultado, pues al escucharse a sí mismo puede comprobar la magnitud del esfuerzo que ha de realizar para que su voz sea de intensidad normal. De este modo se va induciendo al sujeto a que re-evalúe su esfuerzo después de cada ejercicio y a que contraste su percepción con los resultados reales. Finalmente llega a reconocer la necesidad de establecer un nuevo sistema de calibración de su propio esfuerzo; es decir, aprende a reconocer el nivel de esfuerzo que necesita y a aceptar que aunque éste sea grande, su intensidad de voz no llega a ser fuerte, sino normal para los oyentes (aunque él sienta que habla fuerte). Así, al darse cuenta de que la intensidad de su voz suena normal si él la produce con máximo esfuerzo, reestructura de nuevo sus esquemas y programas motores (Ramig et al., 2001b; Liotti et al., 2003).

Otro modo complementario de reforzar esta autoconciencia es mediante la medición diaria de resultados objetivos. El clínico debe cuantificar los resultados obtenidos en cada sesión, e informar al paciente de ellos para que conozca sus logros, se implique más en las tareas y se motive con su línea de progreso. Las variables que son medidas son las que constituyen los objetivos terapéuticos (la intensidad vocal, el tiempo de fonación máxima, el rango tonal) y estas mediciones se repiten varias veces a lo largo de la sesión para compensar la variabilidad de las ejecuciones (Baumgartner et al., 2001). Se ha demostrado que la diaria medición de resultados refuerza por sí misma el aprendizaje del sujeto porque llega a comprender mejor los efectos que va consiguiendo con su esfuerzo (Trail et al., 2005).

La pauta básica de administración del tratamiento consiste en cuatro semanas, durante las cuales se mantienen los mismos objetivos y la misma programación de tareas en bloque; e igualmente se utiliza de forma invariable el mismo tipo de feedback (sobre los parámetros de la ejecución y sobre los resultados globales del ejercicio). Igualmente se estimula sin cesar la motivación del enfermo. Progresivamente se va incrementando la complejidad del material verbal y se van trabajando objetivos múltiples, pero empleando para ellos la misma consiga sencilla ("piense: fuerte, y luego hable fuerte"). Esto es, durante la primera semana, los objetivos se practican en bloque con vocales sostenidas, palabras y frases breves; durante la segunda semana, se utilizan oraciones; en la tercera se trabaja la lectura de párrafos; en la cuarta se transfieren los objetivos antedichos a la conversación, mediante monólogos (descripciones de imágenes) y diálogos (con y sin guión preestablecido) (Liotti et al., 2003; Neel, 2009).

La primera parte de la sesión se debe dedicar a tareas con máximo esfuerzo; en la segunda mitad se hacen ejercicios más variados y si es posible, se eliminan los ejercicios de esfuerzo isométrico. A veces, basta con aumentar la profundidad inspiratoria para que el individuo emita una voz con el máximo esfuerzo fonatorio.

La administración del tratamiento suele ser, en definitiva, intensiva e individual; generalmente con un total de 16 sesiones en un mes, a razón de tres o cuatro sesiones semanales, con una duración de unos 50-60 minutos cada una. Se pueden repetir estos periodos terapéuticos si es necesario.

\section{Factores que más favorecen la generalización del aprendizaje en el Tratamiento Vocal Intensivo (LSVT $\left.{ }^{\circledR}\right)$}

Mediante ensayos clínicos ha quedado demostrada la eficacia de este método para mejorar la voz y mantener los efectos terapéuticos hasta dos años después del tratamiento (Sapir et al., 2002). Igualmente sus resultados han sido contratados con los efectos de otros tipos de 
tratamiento, como los basados en ejercicios respiratorios puros (Ramig et al., 1995), en ejercicios de esfuerzo respiratorio (Baumgartner et al., 2001), o en la lentificación del ritmo del habla (Kleinow et al., 2001), demostrándose su mayor efectividad en todos los modelos experimentales.

¿Por qué este método favorece la generalización de resultados? La razón esencial radica en que es una metodología basada en los fundamentos del aprendizaje motor, dirigida a favorecer la adquisición y la retención de tareas en enfermos que están severamente afectados en lo motor y discretamente afectados en lo cognitivo.

Uno de los factores que más parecen propiciar la generalización de resultados es que el objetivo primordial del tratamiento es fácil de imitar, "respirar hondo, hablar fuerte”. El aumento de intensidad de voz es un acto que está aprendido y automatizado en las estructuras más arcaicas del sistema nervioso central, ligado al contenido emocional de la información, por lo que al sujeto no se le está pidiendo que realice un nuevo comportamiento ni que distorsione el ritmo de habla, sino que use una conducta natural y ancestral (Fox et al., 2002).

Por otro lado, la consigna "hablar más fuerte" repercute globalmente y amplifica de forma proporcional todos los movimientos orofaciales del habla, no sólo los movimientos de laringe (Neel, 2009). Se ha demostrado que esta técnica de entrenamiento vocal actúa de forma similar a las técnicas empleadas para aumentar el trazo de la escritura y la longitud del paso del enfermo parkinsoniano, que al aumentar la amplitud del primer movimiento, simultáneamente se aumentan la amplitud y coordinación del resto de movimientos secuenciales. Así, al empezar el sujeto con un aumento de la profundidad inspiratoria y de la fuerza del cierre glótico, se produce una onda glótica de mayor amplitud al principio de la emisión, que se puede llegar a mantener si el enfermo aprende a autocontrolar este esfuerzo (Baumgartner et al., 2001).

La simplicidad y la redundancia de las tareas de entrenamiento es otra característica del método que facilita el aprendizaje (Schulz et al., 2000b). Para adaptarse al déficit cognitivo leve que suele acompañar a la enfermedad, se evitan las explicaciones largas y el clínico se apoya mucho en la imitación ("haga lo mismo que hago yo"). Por tanto no requiere mucho esfuerzo atencional ni memoria de trabajo, todo lo cual favorece al sujeto con PI y dificultades cognitivas leves tales como lentitud de pensamiento, dificultad para cambiar el criterio del razonamiento, dificultad para cumplir objetivos simultáneos y falta de memoria inmediata (Adams y Page, 2000). Esta simplicidad, junto con las numerosas repeticiones de cada tarea, proporciona la suficiente redundancia en el entrenamiento como para que el sujeto pueda llegar a compensar su lentitud de aprendizaje.

El énfasis en la auto-monitorización del enfermo con PI parece ser otro de los factores clave para conseguir eficacia terapéutica, pues estas personas presentan un déficit "online" de feedback auditivo y propioceptivo, es decir, tienen dificultades para percibir su propia voz de forma instantánea durante la fonación, pero al escucharse en una grabación sí pueden autoanalizar sus características vocales (Kiran et al., 2001; Fox et al., 2002). Es por esto por lo que el clínico constantemente le hace re-evaluar su voz mediante las grabaciones de los ejercicios preguntándole “¿oye la intensidad de su voz, siente el esfuerzo? Esa es la cantidad de esfuerzo que usted necesita al hablar para que la gente le escuche”. De este modo le ayuda a tomar conciencia de que su voz es demasiado suave y de que al hacer el esfuerzo, la consigue colocar en un nivel normal. Como estas personas también presentan otros tipos de déficits, como la dificultad para autoevaluar la amplitud de sus movimientos y ejecutar movimientos automáticos, con el fin de reducir la aceleración o festinación que aparece al ejecutar secuencias motoras, se les entrena con secuencias rítmicas y se les da una clave interna para mantener el ritmo fonorrespiratorio, que es otra instrucción simple: "respire con frecuencia, hable siempre estando lleno de aire".

La pauta de tratamiento intensivo, con muchas repeticiones en bloque dentro de cada sesión y con un intervalo corto entre una sesión y otra, es otra de las condiciones interesantes para compensar las dificultades de aprendizaje motor del enfermo con PI. Adicionalmente, la práctica a solas, en el domicilio, le permite transferir el aumento de intensidad a otras tareas no imitativas como la lectura y habla espontánea; lo cual colabora a que estas personas se vayan sintiendo progresivamente más cómodas en su nuevo nivel de esfuerzo fonatorio, desarrollando así un nuevo esquema corporal y una mayor autoconciencia del acto fonorrespiratorio (Cuadro 1). 
Para terminar cabe resaltar la existencia de estudios que corroboran la generalización de los efectos terapéuticos del LSVT, la cual ha sido valorada y medida mediante diversos tipos de medios instrumentales. Especialmente han sido demostradas las mejorías de los siguientes parámetros:

a. Intensidad vocal. Se ha conseguido aumentarla en todas las tareas entrenadas, y aunque a largo plazo disminuye un poco la ganancia (sobre todo en las tareas más espontáneas como el habla), siempre se mantiene una intensidad por encima de los niveles previos al tratamiento (Ramig et al., 2001a; Spielman et al., 2007).

b. Cierre glótico. Mediante electroglotografía y videoendoscopia se ha evidenciado un cierre glótico más rápido y completo, junto con una mejor definición de los formantes (Hömberg, 1993; Countryman et al., 1997; Fox et al., 2002;). En este sentido se ha demostrado el incremento de tono muscular del tiroaritenoideo en el electromiograma (Ramig et al., 2001a). Es interesante reseñar que este mayor cierre se consigue sin provocar hiperadducción glótica ni hiperfunción fonatoria, sino flexibilizando los movimientos laríngeos y aumentando la variabilidad tonal prosódica (Sapir et al., 2002; Spielman et al., 2003).

c. Actividad motora de labios y lengua. Estudios electromiográficos indican que la repercusión global de los resultados del LSVT se ve reflejada asimismo en la mayor amplitud de los movimientos labiales fonoarticulatorios. Incluso con videofluoroscopia se ha encontrado un incremento de la fuerza lingual durante la fase oral de la deglución. Es decir, se obtienen efectos generalizados no solo sobre la calidad de voz, sino sobre la inteligibilidad del habla y la deglución (Kleinow et al., 2001; El Sharkawi et al., 2002; Neel et al., 2009)

d. Actividad de áreas motoras cerebrales. La tomografía por emisión de positrones (PET) ha puesto de manifiesto que al realizar tareas de habla, aparece una mayor actividad en los centros encefálicos responsables de la automatización de movimientos; junto a estos aparece también una disminución de la actividad en las áreas corticales que no deben participar en tareas motoras ya aprendidas. Todo esto parece indicar que se ha verificado un aprendizaje motor (Liotti et al., 2003; Narayana et al., 2011).

e. También se han utilizado registros perceptuales que han sido llevados a cabo por profesionales no relacionados con el tratamiento (medición a ciegas), así como por el enfermo y sus familiares (Baumgartner et al., 2001).

Los trabajos publicados sobre la medición de los efectos del método LSVT están suponiendo un avance en la historia del tratamiento vocal de las enfermedades neurodegenerativas por todas las razones arriba enumeradas. No obstante para que este tratamiento vocal rinda todos sus beneficios es importante que el individuo con PI esté estable respecto a su medicación, esto es, que se haya alcanzado la dosis óptima y se encuentre en una fase evolutiva sin altibajos. Lógicamente si la enfermedad no ha sido controlada mínimamente y el sujeto está sufriendo crisis de empeoramiento, la rehabilitación vocal no podrá ser tan efectiva. Los enfermos tratados con LSVT se encontraban en estadios II, III y IV de la clasificación de Höen-Yahr, es decir, dentro de una variedad amplia de estadios evolutivos, pero los resultados publicados son más satisfactorios mientras menor sea la etapa en la que se encuentre la enfermedad (Beal et al., 1997).

\section{Conclusiones}

Las condiciones para que se produzca aprendizaje motor vienen dadas por la planificación de objetivos, la programación de tareas y la administración del feedback, todo lo cual consigue crear una nueva imagen mental del movimiento. Existe evidencia científica de que el aprendizaje motor puede ser favorecido en personas con enfermedades neurodegenerativas si se lleva a cabo una programación específica.

Se considera que la efectividad del método LSVT radica en el hecho de que un único objetivo terapéutico (aumentar la intensidad de voz) y una consigna simple ("piense fuerte, hable fuerte"), producen un efecto generalizado en todos los subsistemas de la comunicación oral, de formas que al decirle que hable más fuerte se le induce a hacer movimientos más amplios de respiración, de cierre glótico y de vocalización, con lo que mejora globalmente, no sólo la voz, sino la inteligibilidad del habla y la expresión facial. 
En definitiva, el efecto del método LSVT sobre la voz se produce mediante dos tipos de mecanismos: A) por un lado, al incrementar el input respiratorio y la fuerza de cierre glótico en enfermos con hipofonía, se produce una mejoría en la función glótica, pues al aumentar la amplitud de sus movimientos ondulatorios origina una mejor señal acústica, con más armónoicos que se refuerzan en el tracto vocal; todo lo cual mejora la intensidad sin provocar hiperfunción vocal. Asimismo se produce un aumento del tiempo fonatorio, del rango de la frecuencia fundamental y de la variabilidad de ésta, que se manifiestan con una mejor prosodia, tanto durante la lectura como en el habla espontánea. B) Por otro lado, el aumento de la amplitud de movimientos de la musculatura fonorrespiratoria repercute globalmente sobre el resto del sistema fonatorio y se generaliza, aumentando así la amplitud y coordinación de movimientos faríngeos y orofaciales; es decir, mejora no sólo la voz, sino también otras funciones motoras de la vía aerodigestiva superior, como la deglución, vocalización, fonoarticulación y expresividad facial. El aumento de la expresión, a su vez, mejora el contenido emocional del habla y esto posiblemente contribuya también al progreso observado en la entonación y la prosodia. 
Anexo

\begin{tabular}{|c|c|}
\hline Déficit sensoriomotor & Técnica rehabilitadora \\
\hline $\begin{array}{l}\text { Falta de calibración de la amplitud del } \\
\text { movimiento }\end{array}$ & $\begin{array}{l}\text { Entrenamiento de movimientos muy amplios } \\
\text { (intensidad vocal fuerte) }\end{array}$ \\
\hline $\begin{array}{l}\text { Dificultad para programar movimientos } \\
\text { secuenciales }\end{array}$ & $\begin{array}{l}\text { Repetición de actividades rítmicas dándole claves } \\
\text { externas e internas (feedback) }\end{array}$ \\
\hline Festinación o aceleración en el habla & $\begin{array}{l}\text { Entrenamiento de un ritmo óptimo, determinado } \\
\text { por una clave de coordinación fonorrespiratoria }\end{array}$ \\
\hline $\begin{array}{l}\text { Dificultad para movimientos simultáneos y para } \\
\text { cambiar objetivos }\end{array}$ & $\begin{array}{l}\text { Realizar tareas de secuencias sencillas } \\
\text { (repetición en bloque del mismo movimiento) }\end{array}$ \\
\hline Déficit cognitivo & Técnica compensatoria \\
\hline Dificultad para comprender instrucciones largas & Explicación verbales simples y modelado de tareas \\
\hline Dificultades de retención de nuevas estrategias & $\begin{array}{l}\text { Se le da información redundante: } \\
\text { repetición de tareas y objetivos. } \\
\text { Pauta intensiva de tratamiento } \\
\text { (lo sobre-aprendido es fácil de recordar) }\end{array}$ \\
\hline $\begin{array}{l}\text { Dificultad para atender a varios objetivos y para } \\
\text { cambiar los criterios de pensamiento }\end{array}$ & $\begin{array}{l}\text { Se simplifica y se minimiza el número de } \\
\text { consignas ("hablar fuerte") }\end{array}$ \\
\hline
\end{tabular}

Cuadro 1. Déficits de control motor y cognitivos en el PI, con sus correspondientes enfoques terapéuticos [Adaptado a partir de Hömberg, 1993 y de Fox et al., 2002]. 


\section{Bibliografía}

- Adams, S.G. y Page, A.D. (2000). Effects of selected practice and feedback variables on speech motor learning. J Med Speech Lang Pathol, 8, 215-220.

- Baumgartner, C.A., Sapir, S. y Ramig, L.O. (2001). Voice quality changes following phonatory-respiratory effort treatment (LSVT®) versus respiratory effort treatment for individuals with Parkinson disease. $J$ Voice, 15, 105-114.

- Beal, M.F., Fink, J.S. y Martin, J.B. (1997). Enfermedad de Parkinson y otros trastornos extrapiramidales. En: K.J. Isselbacher, E. Braunwald, J.D. Wilson, J.B. Martin, A.S. Fauci, D.L. Kasper (Eds.), Harrison. Principios de medicina interna (pp. 2620-2626). 13 ${ }^{\mathrm{a}}$ ed. Vol. II. Madrid: McGraw-Hill. Interamericana.

- Beukelman, D.R., Mathy y P., Yorkston, K. (1998). Outcomes measurement in motor speech disorders. En C.M. Frattali (Ed.), Measuring outcomes in speech-language pathology (pp. 334-343). Nueva York-Sttutgart: Thieme.

- Carusso, A.J. y Strand, E.A. (1999). Motor Speech Disorders in Children: Definitions, Background, and A Theoretical Framework. En: A.J. Carusso (Ed.), Clinical Management of Motor Speech Disorders in Children (pp.1-28). New York, Stuttgart: Thieme.

- Clarke, C., Gullaksen, E., McDonald, S. y Lowe, F. (1998). Referral criteria for speech and language therapy assessment of dysphagia caused by idiopatic Parkinson's disease. Acta Neurol Scand, 97, 27-35.

- Constantinescu, G., Theodoros, D., Russell, T., Ward, E., Wilson, S. y Wootton, R. (2011). Treating disordered speech and voice in Parkinson's disease online: a randomized controlled non-inferiority trial. Int. J. Lang. Commun. Disord, 46, 16.

- Countryman, S., Hicks, J., Ramig, L.O. y Smith, M. (1997). Supraglottal hyperadduction in an individual with Parkinson's disease: a clinical treatment note. Am J Speech Lang Path, 6, 74-84.

- El Sharkawi A, Ramig LO, Logeman J, Pauloski B, Rademaker A, Smith C, Pawlas A, Baum, S, y Werner, C. (2002). Swallowing and voice effects of Lee Silverman Voice Treatment (LSVT®): a pilot study. J Neurol Neursurg Psychiatry, 72, 31-36.

- Fisher, B.E., Wu, A.D., Salem, G.J., Song, J., Lin, CH., Yip, J., Cen, S., Gordon, J., Jakowec, M. y Petzinger, G. (2008). The effect of exercise training in improving motor performance and corticomotor excitability in people with early Parkinson's disease. Arch Phys Med Rehabil, 89, 1221-1229.

- Fox, C.M., Ramig, L.O., Ciucci, M.R., Sapir, S., McFarland, D.H. y Farley, B.G. (2006). The Science and Practice of LSVT/LOUD: Neural Plasticity-Principled Approach to Treating Individuals with Parkinson Disease and Other Neurological Disorders. Seminars in Speech \& Language, 27, 283-299.

- Gentile, M., Aucouturier, J., Delong, V. y Sambuis, E. (1994). EMG biofeedback in the treatment of dysarthria. Folia Phoniatr, 46, 188-192.

- Hartelius, L. y Lillvik, M. (2003). Lip and tongue function differently affected in individuals with Multiple Sclerosis. Folia Phoniatr, 55:1-9.

- Hömberg, V (1993). Motor training in the therapy of Parkinson's disease. Neurology, 43, suppl 6, S45-S46.

- Houd, S.C. y Mangolds, V. (1999). Amyotrophic Lateral Sclerosis: a team approach to primary care. Clinical Excellence for Nurse Practitioners, 3, 337-345.

- Howell, S., Tripoliti, E. y Pring T. (2009). Delivering the Lee Silverman Voice Treatment (LSVT) by web camera: a feasibility study. Int J Lang Comm Dis, 44, 287-300.

- Kiran, S. y Larson, C.H. (2001). Effect of duration of pitch-shifted feedback on vocal responses in patients with Parkinson's disease. J Speech Lang Hear Res, 44, 975-987.

- Kleinow, L., Smith, A. y Ramig L.O. (2001). Speech motor stability in IPD: effects of rate and loudness manipulations. J Speech Lang Hear Res, 44, 1041-1051.

- Liotti, M., Ramig, L.O., Vogel, D., Cook, C.I., Ingham, P. y Fox, P.T. (2003). Hypophonia in Parkinson's disease. Neural correlates of voce treatment revealed by PET. Neurology , 60, 432-440.

- Mahler, L.A., Ramig, L.O. y Fox, C. (2009). Intensive Voice Treatment (LSVT (R) LOUD) for Dysarthria Secondary to Stroke. J. Med. Speech-Lang. Pathol, 17, 18. 
- McNeil, M.R., Robin, D.A. y Schmidt, R.A. (1997). Apraxia of speech: definition, differentiation and treatment. En M.R. McNeil (Ed.), Sensorimotor speech disorders (pp. 311-344). New York-Sttutgart: Thieme.

- Mid, I., Dogan, M., Koseoglu, M., Can, G., Sehitoglu, M.A. y Gunal, D.I. (2008). Voice abnormalities and their relation with motor dysfunction in Parkinson's disease. Acta Neurologica Scandinavica, 117, 26-34.

- Narayana, S., Fox, P.T., Zhang, W., Franklin, C., Robin, D.A., Vogel, D. y Ramig, L.O. (2010). Neural Correlates of Efficacy of Voice Therapy in Parkinson's Disease Identified by Performance-Correlation. Hum. Brain Mapp, 31, 15.

- Neel, A.T. (2009). Effects of loud and amplified speech on sentence and word intelligibility in Parkinson disease. J Speech Lang Hear Res, 52, 1021-1033.

- Ramig L.O., Scherer R.C., Klasner E.R., Titze I.R. y Horii, Y. (1990). Acoustic analysis of voice in Amyotrophic Lateral Sclerosis: a longitudinal case study. J Speech Hear Disord, 55, 2-14.

- Ramig, L.O., Countryman, S., Thompson, L.L. y Horii, Y. (1995). Comparison of two forms of intensive speech treatment for Parkinson's disease. J Speech Hear Res, 38, 1232-1251.

- Ramig, L.O., Sapir, S., Countryman, S., Pawlas, A.A., O’Brien, C., Hoehn, M. y Thompson, L.L. (2001a). Intensive Voice Treatment (LSVT ${ }^{\circledR}$ ) for patients with Parkinson's disease: a 2 years follow-up. J Neurosurg Psyciatry, 71, 493-498.

- Ramig, L.O., Sapir, S., Fox, C. y Countryman, S. (2001b). Changes in vocal loudness following Intensive Voice Treatment (LSVT $\left.{ }^{\circledR}\right)$ in individuals with Parkinson's disease: a comparison with untreated patients and normal age-matched controls. Movements Disorders, 16, 79-83.

- Ransmayr, G. (2011). Physical, occupational, speech and swallowing therapies and physical exercise in Parkinson's disease. J. Neural Transm, 118, 9.

- Russell, J.A., Ciucci, M.R., Connor, N.P. y Schallert, T. (2010). Targeted exercise therapy for voice and swallowing in persons with Parkinson disease. Brain Res, 1341, 3-11.

- Sapir S., Ramig, L.O. y Fox, C.M. (2011). Intensive voice treatment in Parkinson's disease: Lee Silverman Voice Treatment. Expert Review of Neurotherapeutics, 11, 815-830.

- Sapir, S., Ramig, L.O., Hoyt, P., Countryman, S., O’Brien, C. y Hoehn, M. (2002). Speech loudness and quality 12 months after Intensive Voice Treatment (LSVT ${ }^{\circledR}$ ) for Parkinson's disease: a comparison with an alternative speech treatment. Folia Phoniatr, 54, 296-303.

- Sapir, S., Ramig, L.O. y Fox, C. (2006). The Lee Silverman Voice Treatment(R) for voice, speech and other orofacial disorders in patients with Parkinson's disease. Future Neurology, 1, 563-570.

- Scherer, R.C. (1991). Physiology of phonation a review of basic mechanics. En C.N. Ford y D.M. Bless (Eds.), Phonosurgery: assessment and surgical management (pp.77-93). Nueva York: Raven Press.

- Schulz, G.M., Sulc, S., Leon, S. y Gilligan, G. (2000). Speech motor learning in Prakinson disease. J Med Speech Lang Pathol, 8, 243-247.

- Spielman, J., Ramig, L.O. y Mahler, L. (2007). Effects of an extended version of the Lee Silverman Voice Treatment on voice and speech in Parkinson's disease. Am J Speech Lang Pathol, 16, 95-107.

- Spielman, J.L., Borod, J.C. y Ramig, L.O. (2003). The effects of Intensive Voice Treatment on facial expressiveness in Parkinson's disease. Preliminary data. Cognitive and Behavioural Neurology, 16, 177-188.

- Square, P.A. (1999). Treatment of developmental apraxia of speech: tactile-kinesthetic, rhythmic, and gestural approaches. En A.J. Caruso y E.A. Strand (Eds.), Clinical management of motor speech disorders in children (pp. 149-185). New York, Stuttgart: Thieme.

- Strand, E.A. y Skinder A. (1999). Treatment of developmental apraxia of speech: integral stimulation methods. En A.J. Caruso y E.A. Strand (Eds.), Clinical management of motor speech disorders in children (pp. 109-148). New York, Stuttgart: Thieme.

- Thompson-Ward, E.C., Murdoch, B.E. y Stokes, P.D. (1997). Biofeedback rehabilitation of speech breathing for an individual with dysarthria. J Med Speech Lang Pathol, 5, 277-288. 
- Weismer, G., Laures, J., Jeng, J., Kent, R., Kent, J. (2000). Effects of speaking rate manipulations on acoustic and perceptual aspects of the dysarthria in Amyotrofic Lateral Sclerosis. Folia Phoniatr, 52, 201-219. 